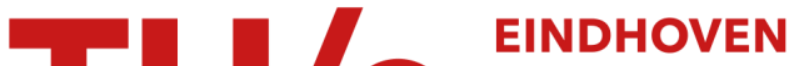 UNIVERSITY OF TECHNOLOGY
}

\section{Dynamic network structure identification with prediction error methods - basic examples}

\section{Citation for published version (APA):}

Dankers, A. G., Hof, Van den, P. M. J., Heuberger, P. S. C., \& Bombois, X. (2012). Dynamic network structure identification with prediction error methods - basic examples. In J. Schoukens, \& R. Bitmead (Eds.), Proceedings of the 16th IFAC Symposium on System Identification (SYSID 2012), July 11-13 2012, Brussels, Belgium (pp. 876-881)

Document status and date:

Published: 01/01/2012

\section{Document Version:}

Accepted manuscript including changes made at the peer-review stage

\section{Please check the document version of this publication:}

- A submitted manuscript is the version of the article upon submission and before peer-review. There can be important differences between the submitted version and the official published version of record. People interested in the research are advised to contact the author for the final version of the publication, or visit the $\mathrm{DOI}$ to the publisher's website.

- The final author version and the galley proof are versions of the publication after peer review.

- The final published version features the final layout of the paper including the volume, issue and page numbers.

Link to publication

\section{General rights}

Copyright and moral rights for the publications made accessible in the public portal are retained by the authors and/or other copyright owners and it is a condition of accessing publications that users recognise and abide by the legal requirements associated with these rights.

- Users may download and print one copy of any publication from the public portal for the purpose of private study or research.

- You may not further distribute the material or use it for any profit-making activity or commercial gain

- You may freely distribute the URL identifying the publication in the public portal.

If the publication is distributed under the terms of Article 25fa of the Dutch Copyright Act, indicated by the "Taverne" license above, please follow below link for the End User Agreement:

www.tue.nl/taverne

Take down policy

If you believe that this document breaches copyright please contact us at:

openaccess@tue.nl

providing details and we will investigate your claim. 


\title{
Dynamic network structure identification with prediction error methods - basic examples
}

\author{
Arne G. Dankers ${ }^{*, 1}$, Paul M.J. Van den Hof ${ }^{* * * *}$, \\ Peter S.C. Heuberger* and Xavier Bombois* \\ * Delft Center for Systems and Control, Delft University of \\ Technology, Mekelweg 2, 2628 CD Delft, The Netherlands (e-mail: \\ a.g.dankers@tudelft.nl) \\ ** Dept. of Electrical Engineering, Eindhoven University of Technology, \\ P.O. Box 513, 5600 MB Eindhoven, The Netherlands
}

\begin{abstract}
:
Modeling of dynamical properties of highly complex and interconnected systems becomes important in different fields of science. When identifying the structure and dynamics of a network of interconnected dynamical systems, including cause-effect relations, there is a tendency to use nonparametric or FIR models of the output error type. In this paper it is shown, and illustrated by some simple examples, that appropriate attention should be given to using flexible noise models, in order to allow consistent identification of the dynamics, while the use of external excitation/probing signals may reduce this need. It is a first step towards using prediction error identification tools to identify the structure of a network.
\end{abstract}

Keywords: System identification; Identifiability; Network topologies; Complex systems; Prediction error methods.

\section{INTRODUCTION}

In the field of system identification one is used to identify a system (and possibly noise dynamics) in a system/measurement/excitation structure that is clearly well defined a priori. One knows up front where the excitation takes place, where causal transfer functions appear, and where noise sources are assumed to be present. Typically one restricts to well-defined a priori chosen structures:

- Open-loop system with possible excitation on the input, and additive noise disturbance on the output;

- The previous situation, but with noisy measurements of the input also (errors-in-variables approach);

- Feedback configuration, with or without external excitation and disturbances at particular locations.

- Scalar (SISO) and/or multivariable (MIMO).

From this standard situation there are two different directions for natural extension of the problem.

(1) If the data generating system is a complex network of interconnected dynamic subsystems, one would like to be able to identify the dynamics of the subsystems.

A conceptually simple solution could be to write the system back to a centralized form and into one of the classical open-loop or closed-loop schemes and apply standard multivariable identification methods. In this situation the structural properties of the MIMO models would become central. Thus new concepts of structured models should be explored, in their use,

1 The work of Arne Dankers is supported in part by the National Science and Engineering Research Council (NSERC) of Canada. suitability and consequences for identification purposes. Some contributions to identification of structured systems can be found in Gudi and Rawlings [2006], Leskens and Van den Hof [2007], Wahlberg et al. [2009], Massioni and Verhaegen [2008].

(2) If the interconnection structure of the subsystems is not known a priori and should be identified also on the basis of measurement data, there appears a problem of structure or topology identification. The basic question then becomes, under which conditions can we identify the interconnection structure of individual subsystems that form an interconnected system.

In this paper we are going to elaborate on this second problem, that seems to have not been addressed very extensively in the identification literature.

Early contributions to this problem date back to Anderson and Gevers [1982], Gevers and Anderson [1981, 1982] who on the basis of the work of Granger [1969] and Caines and Chan [1975], Caines [1976] address the question whether an open-loop or closed-loop structure is present between two measured signals $u$ and $y$. The conclusion was that for particular structures (two measured signals driven by two independent noise sources of similar dimensions), a unique separation of forward (system) and backward (feedback) path could be determined under fairly general conditions. This basically led to the joint i-o approach of closed-loop identification.

More generalized system configurations do not seem to have been addressed in the identification literature. However currently interest has been renewed in trying to detect 
the topology of more complex networks based on measurements of the system (Friedman et al. [2010], Yuan et al. [2010], Sanandaji et al. [2011], Materassi and Innocenti [2010], Materassi et al. [2011] among many others).

In these papers the data generating system is assumed to be described by a node-and-link style of network, as shown in Fig. 1. The goal is to recover the topology using only time records of the variables $w_{i}, i=1, \ldots, L$. The output of each node is:

$$
w_{i}=\sum_{\substack{j=1 \\ j \neq i}}^{L} G_{i j}(q, \theta) w_{j}+v_{i}
$$

where $v_{i}$ a (stochastic) noise disturbance.

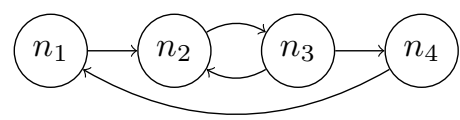

Fig. 1. Node-and-link style network used in topology identification. Each node $n_{i}$ represents a signal $w_{i}$, and arrows represent causal (dynamic) relationships.

Most often nonparametric or FIR output error models are used to model the dynamics of the links $G_{i j}(q, \theta)$ Friedman et al. [2010], Yuan et al. [2010], Sanandaji et al. [2011], Materassi and Innocenti [2010], Materassi et al. [2011]. Materassi and Innocenti [2010] identify a tree-based structure, where in each node an additive noise disturbance is present, and only passive data is taken from the network. Non-causal Wiener filters determine the dynamic components in the tree structure. This work is extended in Innocenti and Materassi [2008], Materassi et al. [2011] towards a more general interconnection structure, and to the use of causal Wiener filters as models. In Sanandaji et al. [2011] a similar structure is considered but FIR models are employed. Sometimes there are assumptions on the presence of external excitation at each node, and the possibility to perform experiments (Timme [2007]).

In this paper we will consider a prediction-error approach to detect the interconnection structure of a dynamical network. The approach will be illustrated using some basic examples. The sensitivity of the approach to the presence of noise sources will also be investigated.

In Section 2 we will sketch the identification approach, in Sections 3 and 4 we will show some of its consequences for a cascaded system topology. In Section 5 a closed loop structure will be discussed. Some discussion on the benefits of external excitation signals will follow in Section 6 .

\section{IDENTIFICATION SETUP}

Consider a data generating system which is a network of nodes as shown in Fig. 1. The output of node $i$ is:

$$
w_{i}=\sum_{\substack{j=1 \\ j \neq i}}^{L} G_{i j}^{0}(q) w_{j}+v_{i}
$$

with $v_{i}$ a stochastic noise disturbance, determined by

$$
v_{i}(t)=H_{i}^{0}(q) e_{i}(t)
$$

where for every $i=1, \ldots, L, H_{i}^{0}$ is a monic stable filter with a stable inverse, $e_{i}$ is a white noise process, and $e_{i}$ and $e_{j}$ are independent for $i \neq j$. The variance of $v_{i}$ is denoted $\sigma_{v_{i}}^{2}$.

The structure of a network is considered as the interconnections between the subsystems of the network. Since the measurements are assumed to have been taken at the outputs of the subsystems, detecting the structure is equivalent to detecting non-zero dynamics between the measurements. If it is decided in some manner that the transfer function from variable $w_{j}$ to variable $w_{i}$ is zero, then the conclusion is that there is no connection between these two nodes, implicitly identifying the topology.

The dynamics will be estimated using the prediction-error method. The one-step ahead predictor is (Ljung [1999]):

$$
\begin{aligned}
\hat{w}_{i}(t \mid t-1, \theta)=H_{i}^{-1}(q, \theta)\left(\sum_{\substack{j=1 \\
j \neq i}}^{L} G_{i j}(q, \theta) w_{j}\right) \\
+\left(1-H_{i}^{-1}(q, \theta)\right) w_{i} .
\end{aligned}
$$

The prediction error is defined as the difference between the measured output and the predictor output:

$$
\varepsilon(t, \theta)=w_{i}(t)-\hat{w}_{i}(t \mid t-1, \theta) .
$$

When adopting a standard least squares identification criterion, under weak assumptions the parameter estimate converges to the minimizing argument $\theta^{*}$ of $\bar{V}_{i}$, where,

$$
\bar{V}_{i}(\theta)=\lim _{N \rightarrow \infty} \frac{1}{N} \sum_{t=0}^{N-1} E\left[\varepsilon_{i}(t, \theta)^{2}\right] .
$$

Our investigation will consist of studying some very simple networks, and studying what the effects of certain assumptions are on the ability of the proposed method to recover the network topology. The first simple network considered will be an open-loop cascaded network. The second will be a closed loop network.

\subsection{Wiener Filter Approach}

In Materassi et al. [2011] this problem has been analyzed on the basis of (causal) Wiener filter estimates of the transfers functions $G_{i j}$.

The optimal causal Wiener filter $W(z)$ that estimates the transfer function between $u$ and $y$ is defined by the so called Wiener-Hopf equations (Benesty [2008]):

$$
R_{u y}(\tau)=\sum_{t=0}^{\infty} w(t) R_{y}(\tau-k), \quad \tau=0,1,2, \ldots,
$$

where $R_{u y}$ and $R_{y}$ are the cross-correlation between $u$ and $y$ and the autocorrelation of $y$ respectively.

In the appendix it is shown that in the prediction-error framework the optimal causal Wiener filter is equivalent to the asymptotically optimal FIR model estimate, with the model order tending to infinity. We will proceed using the prediction error framework, and the analysis tools available in this framework, but all the results apply to the causal Wiener filter approach as well. 


\section{A OPEN-LOOP CASCADE EXAMPLE - OUTPUT ERROR MODELS}

Consider a data generating system to be composed of a concatenation of two systems, according to:

$$
\begin{aligned}
& w_{1}=G_{1 r}^{0} r+v_{1} \\
& w_{2}=G_{21}^{0} w_{1}+v_{2},
\end{aligned}
$$

with $G_{1 r}^{0}, G_{21}^{0}$ proper transfer functions.

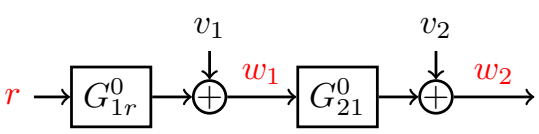

Fig. 2. Cascade system with measured signals in red.

We will identify this system, but without any a priori information on its structure. We will only use the information that we have 3 measured signals $w_{1}, w_{2}, r$, with $r$ being persistently exciting of a sufficiently high order.

In this section, we will focus our attention to identifying the dynamics between measurement $w_{1}$ as the output, and measurements $w_{2}$ and $r$ as the inputs using the OutputError (OE) model structure. For this model structure $H_{i}(\theta)$ in (3) is equal to 1 , resulting in the predictor

$$
\hat{w}_{1}(t \mid t-1, \theta)=G_{12}(q, \theta) w_{2}+G_{1 r}(q, \theta) r .
$$

Both $w_{2}$ and $r$ are included as inputs since it is not known a priori that $w_{2}$ in fact does not causally affect $w_{1}$. Since we are using a causal predictor, we expect the estimated $G_{12}(q, \theta)$ between $w_{1}$ and $w_{2}$ to be zero, and $G_{1 r}(q, \theta)$ between $w_{1}$ and $r$ to be non zero.

From Section 2 the asymptotical estimates of $G_{12}(q, \theta)$ and $G_{1 r}(\theta)$ are determined by the minimizing argument of (4). Using (7) the expression for the prediction error is

$$
\begin{aligned}
& \varepsilon_{1}(t, \theta)=w_{1}(t)-\hat{w}_{1}(t \mid t-1, \theta) \\
& =w_{1}(t)-G_{12}(\theta) w_{2}(t)-G_{1 r}(\theta) r(t) \\
& =\left(G_{1 r}^{0}-G_{12}(\theta) G_{21}^{0} G_{1 r}^{0}-G_{1 r}(\theta)\right) r(t) \\
& \quad+\left(1-G_{12}(\theta) G_{21}^{0}\right) H_{1}^{0} e_{1}(t)-G_{12}(\theta) H_{2}^{0} e_{2}(t)
\end{aligned}
$$

Using (8) and Parseval's theorem the identification criterion (4) can be expressed as

$$
\begin{aligned}
\bar{V}(\theta) & =\frac{1}{2 \pi} \int_{-\pi}^{\pi} \mid G_{1 r}^{0}\left(e^{i \omega}\right)-G_{12}\left(e^{i \omega}, \theta\right) G_{21}^{0}\left(e^{i \omega}\right) G_{1 r}^{0}\left(e^{i \omega}\right) \\
& -\left.G_{1 r}\left(e^{i \omega}, \theta\right)\right|^{2} \Phi_{r}(\omega) \\
+ & \left|\left(1-G_{12}\left(e^{i \omega}, \theta\right) G_{21}^{0}\left(e^{i \omega}\right)\right) H_{1}^{0}\left(e^{i \omega}\right)\right|^{2} \sigma_{e_{1}}^{2} \\
& +\left|G_{12}\left(e^{i \omega}, \theta\right) H_{2}^{0}\left(e^{i \omega}\right)\right|^{2} \sigma_{e_{2}}^{2} \mathrm{~d} \omega
\end{aligned}
$$

where $\Phi_{r}$ is the power spectral density of $r$ and $\sigma_{e_{1}}^{2}$ and $\sigma_{e_{2}}^{2}$ are the variances of the noise terms $e_{1}$ and $e_{2}$.

Note that $\bar{V}(\theta)$ is a sum of three positive terms. This separation into three terms is possible due to the assumption that $r, e_{1}$ and $e_{2}$ are independent.

Next we will consider three different cases:

- $G_{1 r}^{0}, G_{21}^{0}$ are strictly proper and $v_{1}, v_{2}$ are white.

- $G_{1 r}^{0}, G_{21}^{0}$ are strictly proper and $v_{1}, v_{2}$ are colored.

- $G_{1 r}^{0}, G_{21}^{0}$ are not strictly proper and $v_{1}, v_{2}$ are white.

\subsection{Case 1: $G_{1 r}^{0}, G_{21}^{0}$ strictly proper and $v_{1}, v_{2}$ white}

In this case $H_{1}=H_{2}=1$. Since $G_{21}^{0}(q)$ is strictly proper, the smallest value the $e_{1}$-dependent term in (9) can achieve is 1 . The smallest value the $r$ and $e_{2}$-dependent terms can achieve is 0. Each term achieves its minimum when

$$
\begin{aligned}
& G_{12}\left(\theta^{*}\right)=0 \\
& G_{1 r}\left(\theta^{*}\right)=G_{1}
\end{aligned}
$$

provided that these choices are present in the considered model set, and provided that $\sigma_{v_{1}}^{2}, \sigma_{v_{2}}^{2}>0$.

If $\sigma_{v_{1}}^{2}=\sigma_{v_{2}}^{2}=0$, then we have the situation that $G_{1 r}(\theta)=G_{1 r}^{0}-G_{12}(\theta) G_{21}^{0} G_{1 r}^{0}$ minimizes $(9)$, and $G_{12}(\theta)$ is free. In other words, non-unique estimates are obtained.

Considering all possible situations of values of $\sigma_{v_{1}}, \sigma_{v_{2}}$ we obtain the results as indicated in the following table:

\begin{tabular}{|c||c|c|c|c|}
\hline$\sigma_{v_{1}}$ & $>0$ & $>0$ & $=0$ & $=0$ \\
$\sigma_{v_{2}}$ & $>0$ & $=0$ & $>0$ & $=0$ \\
\hline \hline$G_{12}\left(\theta^{*}\right)$ & 0 & 0 & 0 & non-unique \\
$G_{1 r}\left(\theta^{*}\right)$ & $G_{1 r}^{0}$ & $G_{1 r}^{0}$ & $G_{1 r}^{0}$ & non-unique \\
\hline
\end{tabular}

Table 1.Asymptotic results in Case 1 (strictly proper transfers and white noises).

We can conclude that as long as one of the two noise sources is present, the dynamics is consistently estimated, and the correct structure is identified.

\subsection{Case 2: $G_{1 r}^{0}, G_{21}^{0}$ strictly proper and $v_{1}, v_{2}$ colored}

In this case $H_{1}^{0}$ and $H_{2}^{0}$ are not equal to 1 . When minimizing the power of $(9)(9)$, the $e_{1}$-dependent term is not necessarily minimized for $G_{12}\left(\theta^{*}\right)=0$.

Here, $G_{12}\left(\theta^{*}\right)$ will be such that it minimizes the sum of the two noise terms, and $G_{1 r}\left(\theta^{*}\right)$ is then adjusted to equate the first term to 0 . As a result $G_{12}\left(\theta^{*}\right)$ will be dependent on the relation between the noise powers $\sigma_{v_{1}}^{2}$ and $\sigma_{v_{2}}^{2}$. Therefore it will generally be not consistent. The estimate becomes consistent though if $\sigma_{v_{1}}^{2}=0$, since then the $e_{2}$-dependent term will force $G_{12}(\hat{\theta})$ to become 0 .

\begin{tabular}{|c||c|c|c|c|}
\hline$\sigma_{v_{1}}$ & $>0$ & $>0$ & $=0$ & $=0$ \\
$\sigma_{v_{2}}$ & $>0$ & $=0$ & $>0$ & $=0$ \\
\hline \hline$G_{12}\left(\theta^{*}\right)$ & biased & biased & 0 & non-unique \\
$G_{1 r}\left(\theta^{*}\right)$ & biased & biased & $G_{1 r}^{0}$ & non-unique \\
\hline
\end{tabular}

Table 2. Asymptotic results in Case 2 (strictly proper transfers and colored noises).

As a conclusion we can observe that in general the estimation results will be biased, which will limit the determination of the topology. Only in the situation of absence of the noise source $v_{1}$, the structure is correctly retrieved.

3.3 Case 3: $G_{1 r}^{0}, G_{21}^{0}$ not strictly proper and $v_{1}, v_{2}$ white

In this case again $H_{1}^{0}=H_{2}^{0}=1$. If $G_{21}^{0}$ is not strictly proper, then a choice $G_{12}\left(\theta^{*}\right)=\left(G_{21}^{0}\right)^{-1}$ would actually allow the $e_{1}$-dependent term in (9) to become 0 . However since $G_{12}(\theta)$ appears also in the $e_{2}$-term, the estimate $G_{12}(\hat{\theta})$ will generally be biased, and dependent on the relation between the two noise powers of $e_{1}$ and $e_{2}$. 


\begin{tabular}{|c||c|c|c|c|}
\hline$\sigma_{v_{1}}$ & $>0$ & $>0$ & $=0$ & $=0$ \\
$\sigma_{v_{2}}$ & $>0$ & $=0$ & $>0$ & $=0$ \\
\hline \hline$G_{12}\left(\theta^{*}\right)$ & biased & $\left(G_{21}^{0}\right)^{-1}$ & 0 & non-unique \\
$G_{1 r}\left(\theta^{*}\right)$ & biased & 0 & $G_{1 r}^{0}$ & non-unique \\
\hline
\end{tabular}

Table 3. Asymptotic results in Case 3 (proper transfers and white noises).

We can conclude that in general the estimation results will be biased, which will limit the determination of the correct topology. Only in the situation of absence of the noise source $e_{1}$, the structure is correctly retrieved.

\subsection{Discussion}

This example shows that the correct structure can be retrieved from the measured signals, in the situation that the dynamic links are strictly proper, and the noise is white (i.e. the noise is correctly modeled with an OE model). In the prediction error framework, this latter condition is formulated as: the system should be included in the model set. Whereas in classical open-loop system identification it is possible to consistently identify plant transfers with output error models, even if the measured signals are disturbed by colored noises, apparently this property disappears in the structure identification problem.

\section{A OPEN-LOOP CASCADE EXAMPLE - PARAMETERIZED NOISE MODELS}

A partial remedy to the problem indicated in the previous section is the inclusion of appropriate noise models in the considered model structure, leading to the prediction error

$$
\begin{aligned}
\varepsilon_{1}(t, \theta)= & H_{1}(\theta)^{-1}\left[\left(G_{1 r}^{0}-G_{12}(\theta) G_{21}^{0} G_{1 r}^{0}-G_{1 r}(\theta)\right) r(12)\right. \\
& \left.+\left(1-G_{12}(\theta) G_{21}^{0}\right) H_{1}^{0} e_{1}-G_{12}(\theta) H_{2}^{0} e_{2}\right] .
\end{aligned}
$$

Consider the same three cases as in the previous section.

\subsection{Case 1: $G_{1 r}^{0}, G_{21}^{0}$ strictly proper and $v_{1}, v_{2}$ white}

By looking at (12) we can see that the smallest value the $e_{1}$-dependent term in $\bar{V}(\theta)$ can achieve is $\sigma_{e_{1}}^{2}$. This minimum is achieved when $H_{1}(\theta)=1-G_{12}(\theta) G_{21}^{0}$.

When $\sigma_{e_{2}} \neq 0, G_{12}(\theta)$ is forced to zero by the $e_{2}$-dependent term in $V(\theta)$.

However, when $\sigma_{e_{2}}^{2}=0$, we have

$$
\begin{aligned}
H_{1}(\theta) & =1-G_{12}(\theta) G_{21}^{0} \\
G_{1 r}(\theta) & =G_{1 r}^{0}-G_{12}(\theta) G_{21}^{0} G_{1 r}^{0},
\end{aligned}
$$

where $G_{12}(\theta)$ is free. The results are tallied in Table 4 .

\begin{tabular}{|c||c|c|c|c|}
\hline$\sigma_{v_{1}}$ & $>0$ & $>0$ & $=0$ & $=0$ \\
$\sigma_{v_{2}}$ & $>0$ & $=0$ & $>0$ & $=0$ \\
\hline \hline$G_{12}\left(\theta^{*}\right)$ & 0 & non-unique & 0 & non-unique \\
$G_{1 r}\left(\theta^{*}\right)$ & $G_{1 r}^{0}$ & non-unique & $G_{1 r}^{0}$ & non-unique \\
\hline
\end{tabular}

Table 4. Asymptotic results in Case 1 (strictly proper transfers and white noises) - with parameterized noise models.

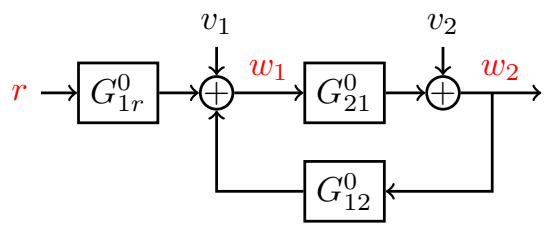

Fig. 3. Example closed-loop system.

Compared to Case 1 in the previous section, it appears that adding noise models in this case actually leads to less powerful results. It seems that the extra flexibility in the model structure is a hindrance.

\subsection{Case 2: $G_{1 r}^{0}, G_{21}^{0}$ strictly proper and $v_{1}, v_{2}$ colored}

The reasoning in this case is exactly the same is in Case 1, with the same results as in Table 4 .

Compared to Case 2 in Section 3.2 it appears that modeling the noise is beneficial since it leads to unbiased estimates if both $\sigma_{e_{1}}^{2}$ and $\sigma_{e_{2}}^{2}$ are nonzero. In the other cases, there is no advantage to modeling the noise.

The results for Case 3, not strictly proper transfer functions $G_{1 r}^{0}$ and $G_{21}^{0}$, however stays the same as before. In this case no correct structure is identified, unless $\sigma_{e_{1}}^{2}=0$.

\subsection{Discussion}

The results of this example show that again, the correct structure can be retrieved from the measured signals in the situation that the transfer functions are strictly proper, and the noise sources are correctly modeled. There is an exception however when the noise in the data generating system is white, the added flexibility in the model structure actually inhibits the possibility of consistent estimates. In a normal open loop system identification setting the added flexibility is not a problem, apparently this property disappears in the structure identification problem.

\section{A CLOSED-LOOP EXAMPLE}

By adding a third interconnection to the data generating system, we can move to a closed-loop structure, as depicted in Fig. 3. The system equations only have one additional term, in comparison to the cascade example from the previous section:

$$
\begin{aligned}
& w_{1}=G_{1 r}^{0} r+G_{12}^{0} w_{2}+v_{1}, \\
& w_{2}=G_{21}^{0} w_{1}+v_{2} .
\end{aligned}
$$

With the sensitivity function:

$$
S^{0}:=\frac{1}{1+G_{12}^{0} G_{21}^{0}}
$$

the system equations read:

$$
\begin{aligned}
& w_{1}=G_{1 r}^{0} S^{0} r+S^{0} v_{1}-G_{12}^{0} S^{0} v_{2} \\
& w_{2}=G_{21}^{0} G_{1 r}^{0} S^{0} r+G_{21}^{0} S^{0} v_{1}+S^{0} v_{2} .
\end{aligned}
$$

Using the models with parameterized noise models, the prediction error for signal $w_{1}$ is: 


$$
\begin{aligned}
& \varepsilon_{1}(t, \theta)=H_{1}(\theta)^{-1}\left[w_{1}(t)-G_{12}(\theta) w_{2}(t)-G_{1 r}(\theta) r(t)\right] \\
& \quad=H_{1}(\theta)^{-1}\left[\left(G_{1 r}^{0} S^{0}-G_{12}(\theta) G_{21}^{0} G_{1 r}^{0} S^{0}-G_{1 r}^{0}(\theta)\right) r+\right. \\
& \left.\quad+S^{0}\left(1-G_{12}(\theta) G_{21}^{0}\right) H_{1}^{0} e_{1}-S^{0}\left(G_{12}^{0}+G_{12}(\theta)\right) H_{2}^{0} e_{2}\right]
\end{aligned}
$$

For strictly proper transfers $G_{1 r}^{0}, G_{21}^{0}, G_{12}^{0}$ and colored noises $v_{1}, v_{2}$ we now arrive at the following results using the same reasoning as before. If we fix $H(\theta)=1$ (i.e. $\mathrm{OE}$ model structure) then:

\begin{tabular}{|c||c|c|c|c|}
\hline$\sigma_{v_{1}}$ & $>0$ & $>0$ & $=0$ & $=0$ \\
$\sigma_{v_{2}}$ & $>0$ & $=0$ & $>0$ & $=0$ \\
\hline \hline$G_{12}\left(\theta^{*}\right)$ & biased & non-unique & $-G_{12}^{0}$ & non-unique \\
$G_{1 r}\left(\theta^{*}\right)$ & biased & non-unique & $G_{1 r}^{0}$ & non-unique \\
\hline
\end{tabular}

Table. 5 Asymptotic results in Case 2 (strictly proper transfers and colored noises) - with FIR/OE models.

If we do not fix $H_{1}(\theta)=1$ then:

\begin{tabular}{|c||c|c|c|c|}
\hline$\sigma_{v_{1}}$ & $>0$ & $>0$ & $=0$ & $=0$ \\
$\sigma_{v_{2}}$ & $>0$ & $=0$ & $>0$ & $=0$ \\
\hline \hline$G_{12}\left(\theta^{*}\right)$ & $-G_{12}^{0}$ & non-unique & $-G_{12}^{0}$ & non-unique \\
$G_{1 r}\left(\theta^{*}\right)$ & $G_{1 r}^{0}$ & non-unique & $G_{1 r}^{0}$ & non-unique \\
\hline
\end{tabular}

Table. 6 Asymptotic results in Case 2 (strictly proper transfers and colored noises) - with parameterized noise models.

In the current situation the estimate $G_{12}\left(\theta^{*}\right)=-G_{12}^{0}$ and $G_{1 r}\left(\theta^{*}\right)=G_{1 r}^{0}$ corresponds to the correct structure. It is correctly identified in the situation that all noise sources are present, only when parameterized noise models are applied. With OE-models, a bias occurs in the estimates. A second opportunity for estimating the correct structure is when there is no noise present on the considered signal $\left(\sigma_{v_{1}}=0\right)$.

\section{POTENTIAL BENEFIT OF EXCITATION SIGNALS}

In our examples and analysis presented so far we have not made explicit use of the fact that the excitation signal $r$ is at our disposal for probing the network. Actually in all the results presented in the previous sections, $r$ is actually handled as just any other node signal.

However when we have an external probing signal available for probing the network, then this can provide additional information. Actually this phenomenon comes down to inserting the prior knowledge that the particular node signal only acts as input in the network and not as output.

In closed-loop identification the benefit of excitation signals is well known (Van den Hof [1998], Forssell and Ljung [1999]). It allows us to identify plant dynamics without having the necessity to model all noises correctly, through indirect methods. In a related phrase, it allows us to apply instrumental variable types of techniques, where the external excitation signals can act as external (instrumental) signals that are uncorrelated to all noise sources in the network (Gilson and Van den Hof [2005]). In order to utilize this in a network identification problem, a different model structure and a different identification criterion has to be considered.
As an example we use the closed-loop situation of Section 5 , and we consider the problem of identifying the transfer $G_{21}(\theta)$.

Consider now the predictor

$$
\hat{w}_{2}(t \mid t-1, \theta)=G_{21}(\theta) w_{1}
$$

which means that we have removed the information from the excitation signal $r$.

The prediction error then becomes:

$$
\begin{aligned}
\varepsilon_{2}(t, \theta)= & \left(G_{1 r}^{0} G_{21}^{0} S-G_{21}(\theta) G_{1 r}^{0} S\right) r+ \\
& +S\left(G_{21}^{0}-G_{21}(\theta)\right) v_{1}+S\left(1+G_{21}(\theta) G_{12}^{0}\right) v_{2} .
\end{aligned}
$$

Applying an instrumental variable (IV) method for estimation now comes down to setting:

$\hat{\theta}=\operatorname{sol}_{\theta} \lim _{N \rightarrow \infty} \frac{1}{N} \sum_{t=0}^{N-1} \varepsilon_{2}(t, \theta) r(t-k)=0 \quad$ for $k=1,2,3 \ldots$

If $r$ is an external excitation signal, and uncorrelated with the noise signals $v_{1}, v_{2}$, the solution will be determined by

$$
\left(G_{1 r}^{0} G_{21}^{0} S-G_{21}(\hat{\theta}) G_{1 r}^{0} S\right)=0
$$

implying that $G_{21}(\hat{\theta})=G_{21}^{0}$. This correct result is obtained without any modeling of the (colored) noises $v_{1}$ and $v_{2}$.

It is conjectured that this mechanism can provide appropriate information for detecting the network structure, while refraining from extensive noise modeling. Results of this approach will be presented in future work.

\section{CONCLUSIONS}

In this paper we have touched upon the problem of identifying the network topology of a network of dynamical systems, using classical identification tools, i.e. prediction error methods. FIR/Output error models or equivalently causal Wiener filter models, that are often used for this purpose, are shown to lead to biased results for the dynamic transfers in the network, if the noise contributions are non-white. And biased model estimates seem to be a weak basis for detecting the network structure. A partial solution exists in the use of parameterized noise models. The results of this have been illustrated in two simple examples. This idea is further investigated in the upcoming paper Dankers et al. [2012]

Additionally the particular role of external excitation signals has been discussed, showing the opportunity to identify the network structure while circumventing parameterized noise modeling. This idea is further investigated in the upcoming paper Van den Hof et al. [2012]

The results presented here seem to be in line with the use of so-called Granger filters as presented in the recent work of Materassi et al. [2011], where use seems to be made of ARX (noise) model structures.

\section{APPENDIX}

Proposition 1. Let the prediction model (3) for the $i$ th node be the FIR predictor: 


$$
\hat{w}_{i}(t \mid t-1, \theta)=\sum_{\substack{j=1 \\ j \neq i}}^{L} G_{i j}(q, \theta) w_{j}=\sum_{\substack{j=1 \\ j \neq i}}^{L} W_{j} \theta_{i j}
$$

where $G_{i j}(q, \theta)=\sum_{k=0}^{n_{b}} \theta_{i j}(k) q^{-k}$ and

$$
W_{j}=\left[\begin{array}{cccc}
w_{j}(0) & w_{j}(-1) & \ldots & w_{j}\left(-n_{b}\right) \\
w_{j}(1) & w_{j}(0) & \ldots & w_{j}\left(-n_{b}+1\right) \\
\vdots & \vdots & & \vdots \\
w_{j}(N-1) & w_{j}(N-2) & \ldots & w_{j}\left(N-1-n_{b}\right)
\end{array}\right]
$$

Suppose that all the signals $w_{i}$ are persistently exciting (i.e. each matrix $W_{i}$ has full rank for any $n_{b}$ ). Moreover, assume that each signal $w_{i}$ contains an external excitation that is independent of the other signals $w_{j}, j \neq i$. Then the asymptotically optimal $\hat{\theta}_{i}$ is a MISO causal wiener filter.

Proof. Due to the conditions assumed on the signals $w_{i}$, the matrix $W=\left[\begin{array}{lll}W_{1} & \ldots & W_{L}\end{array}\right]$ has full rank for any value of $n_{b}$. The optimal $\theta$ satisfies the equations:

$$
\left(W^{T} W\right) \hat{\theta}=W^{T} w_{1}
$$

taking the expected value, and letting $N \rightarrow \infty$ results in:

$$
\lim _{N \rightarrow \infty} \frac{1}{N} \mathbb{E}\left[\left(W^{T} W\right) \theta\right]=\lim _{N \rightarrow \infty} \frac{1}{N} \mathbb{E}\left[W^{T} w_{1}\right]
$$

which in turn results in

$$
\left[\begin{array}{ccc}
R_{w_{2}, w_{2}} & \ldots & R_{w_{2}, w_{L}} \\
\vdots & & \vdots \\
R_{w_{L}, w_{2}} & \ldots & R_{w_{L}, w_{L}}
\end{array}\right]\left[\begin{array}{c}
\hat{\theta}_{12} \\
\vdots \\
\hat{\theta}_{1 L}
\end{array}\right]=\left[\begin{array}{c}
\breve{R}_{w_{2}, w_{1}} \\
\vdots \\
\breve{R}_{w_{L}, w_{1}}
\end{array}\right]
$$

where

$$
\begin{aligned}
R_{w_{i}, w_{j}} & =\left[\begin{array}{ccc}
R_{w_{i}, w_{j}}(0) & R_{w_{i}, w_{j}}(1) & \ldots \\
R_{w_{i}, w_{j}}(1) & R_{w_{i}, w_{j}}(0) & \ldots \\
\vdots & \vdots & \ddots
\end{array}\right] \\
\breve{R}_{w_{i}, w_{1}} & =\left[\begin{array}{c}
R_{w_{i}, w_{1}}(0) \\
R_{w_{i}, w_{1}}(1) \\
\vdots
\end{array}\right]
\end{aligned}
$$

where $R_{x, y}(\tau)$ is the cross-correlation function between the signals $x$ and $y$.

These are the famous Wiener-Hopf Equations, and their solution is the multi-input, single-output Wiener Filter Benesty [2008].

\section{REFERENCES}

B. D. O. Anderson and M. R. Gevers. Identifiability of linear stochastic systems operating under linear feedback. Automatica, 18(2):195-213, 1982.

J. Benesty, editor. Springer Handbook of Speech Processing. Springer, 2008.

P. E. Caines. Weak and strong feedback free processes. IEEE Transactions on Automatic Control, 21:737-739, 1976.

P. E. Caines and C. W. Chan. Feedback between stationary stochastic processes. IEEE Transactions on Automatic Control, 20(4):498-508, 1975.

A. Dankers, P. M. J. Van den Hof, P. S. C. Heuberger, and X. Bombois. Dynamic network identification using the direct prediction error method. In Proceedings of 51 IEEE Conference on Decision and Control, 2012. submitted.
U. Forssell and L. Ljung. Closed-loop identification revisited. Automatica, 35(7):1215-1241, 1999.

J. Friedman, T. Hastie, and R. Tibshirani. Applications of the lasso and grouped lasso to the estimation of sparse graphical models. unpublished, 2010. URL

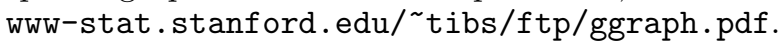

M. R. Gevers and B. D. O. Anderson. On jointly stationary feedback-free stochastic-processes. IEEE Transactions on Automatic Control, 27(2):431-436, 1982.

M. R. Gevers and B. D. O Anderson. Representing of jointly stationary stochastic feedback processes. International Journal of Control, 33(5):777-809, 1981.

M. Gilson and P. M. J. Van den Hof. Instrumental variable methods for closed-loop system identification. Automatica, 41(2):241-249, 2005.

C. W. J. Granger. Investigating causal relations by econometric models and cross-spectral methods. Econometrica, 37(3):424-438, 1969.

R.D. Gudi and J.B. Rawlings. Identification for decentralized model predictive control. AIChE Journal, 52(6): 2198-2210, 2006.

G. Innocenti and D.W. Materassi. Topological properties in identification and modeling techniques. In Proc. 17th IFAC World Congress, pages 15387-15392, Seoul,South Korea, 6-11 July 2008.

M. Leskens and P. M. J. Van den Hof. Closed-loop identification of multivariable processes with part of the inputs controlled. Int. J. Control, 80(10):1552-1561, 2007.

L. Ljung. System Identification: Theory for the User. Prentice-Hall, Englewood Cliffs, NJ, 1999.

P. Massioni and M. Verhaegen. Subspace identification of circulant systems. Automatica, 44(11):2825-2833, 2008.

D. Materassi and G. Innocenti. Topological identification in networks of dynamical systems. IEEE Transactions on Automatic Control, 55(8):1860-1871, 2010.

D. Materassi, M.V. Salapaka, and L Giarrè. Relations between structure and estimators in networks of dynamical systems. In Proceedings of 50th IEEE Conference on Decision and Control, Orlando, USA, 2011.

B. M. Sanandaji, T. L. Vincent, and M. B. Wakin. Exact topology identification of large-scale interconnected dynamical systems from compressive observations. In Proceedings of American Control Conference, pages 649656, San Francisco, CA, USA, 2011.

M. Timme. Revealing network connectivity from response dynamics. Physical Review Letters, 98(22), 2007.

P. M. J. Van den Hof. Closed-loop issues in system identification. Annual Reviews in Control, 22:173-186, 1998.

P. M. J. Van den Hof, A. Dankers, P. S. C. Heuberger, and X. Bombois. Identification in dynamic networks with known interconnection topology. In Proceedings of 51 IEEE Conference on Decision and Control, 2012. submitted.

B. Wahlberg, H. Hjalmarsson, and J. Martensson. Variance results for identification of cascade systems. Automatica, 45:1443-1448, 2009.

Y. Yuan, S. G.-B. Stan, S. Warnick, and J. Gonçalves. Robust dynamical network reconstruction. In 49th IEEE Conference on Decision and Control, pages 810815, Atlanta, GA, USA, 2010. 